TAIWANESE JOURNAL OF MATHEMATICS

Vol. 11, No. 4, pp. 1221-1235, September 2007

This paper is available online at http://www.math.nthu.edu.tw/tjm/

\title{
OSCILLATION THEOREMS RELATED TO AVERAGING TECHNIQUE FOR SECOND ORDER NONLINEAR NEUTRAL DIFFERENTIAL EQUATIONS
}

\author{
Zhiting Xu
}

\begin{abstract}
Some oscillation theorems are established by the averaging technique for the second order nonlinear neutral delay differential equation

$$
\begin{gathered}
\left(r(t)\left|x^{\prime}(t)\right|^{\gamma-1} x^{\prime}(t)\right)^{\prime}+q_{1}(t)\left|y\left(t-\sigma_{1}\right)\right|^{\alpha-1} y\left(t-\sigma_{1}\right) \\
+q_{2}(t)\left|y\left(t-\sigma_{2}\right)\right|^{\beta-1} y\left(t-\sigma_{2}\right)=0, \quad t \geq t_{0},
\end{gathered}
$$

where $x(t)=y(t)+p(t) y(t-\tau), \tau, \sigma_{1}$ and $\sigma_{2}$ are nonnegative constants, $\alpha, \beta$ and $\gamma$ are positive constants, and $r, p, q_{1}, q_{2} \in C\left(\left[t_{0}, \infty\right), \mathbb{R}\right)$. The results obtained here essentially improve some known results in the literature. In particular, two interesting examples that point out the applications of our results are also included.
\end{abstract}

\section{INTRODUCTION}

In this paper, we study the problem of oscillation of the second order nonlinear neutral delay differential equation

$$
\begin{aligned}
& \left(r(t)\left|x^{\prime}(t)\right|^{\gamma-1} x^{\prime}(t)\right)^{\prime}+q_{1}(t)\left|y\left(t-\sigma_{1}\right)\right|^{\alpha-1} y\left(t-\sigma_{1}\right) \\
& \quad+q_{2}(t)\left|y\left(t-\sigma_{2}\right)\right|^{\beta-1} y\left(t-\sigma_{2}\right)=0, \quad t \geq t_{0},
\end{aligned}
$$

where $x(t)=y(t)+p(t) y(t-\tau)$.

In what follows we will assume that

(A1) $\tau, \sigma_{1}$ and $\sigma_{2}$ are nonnegative constants, $\alpha, \beta$ and $\gamma$ are positive constants with $0<\alpha<\gamma<\beta$;

Received August 25, 2005, accepted February 23, 2006.

Communicated by Sze-Bi Hsu.

2000 Mathematics Subject Classification: 34C10, 34C15.

Key words and phrases: Oscillation, Neutral differential equation, Nonlinear, Averaging technique, Second order. 
(A2) $r, q_{1}, q_{2} \in C\left(\left[t_{0}, \infty\right), \mathbb{R}^{+}=(0, \infty)\right)$, and $\int^{\infty} r^{-1 / \gamma}(s) d s=\infty$;

(A3) $p \in C\left(\left[t_{0}, \infty\right), \mathbb{R}\right)$, and $-1<p_{0} \leq p(t) \leq 1, p_{0}$ constant.

Our attention is restricted to those solutions of (1.1) which exist on some half line $\left[t_{x}, \infty\right)$ with $\sup \{|y(t)|: t \geq T\}>0$ for any $T \geq t_{x}$, and satisfy (1.1). We make the standing hypothesis that (1.1) does possess such solution [11]. As usual, a solution of (1.1) is said to be oscillatory if the set of its zeros is unbounded from above, otherwise it is called nonoscillatory. (1.1) is called oscillatory if all of its solutions are oscillatory.

We note that second order neutral delay differential equations are used in many fields such as vibrating masses attached to an elastic bar and some variational problems, etc., see, for example [11].

In the last decades, there has been an increasing interest in obtaining sufficient conditions for the oscillation and /or nonoscillation of second order linear and nonlinear neutral delay differential equations (see, for example, 1-3,5,7-10,14,15,1721,24 and the references therein). For the second order neutral delay differential equation

$$
[y(t)+p(t) y(t-\tau)]^{\prime \prime}+q(t) f(y(t-\sigma))=0 .
$$

To the best of our knowledge, almost all of the known results obtained for (1.2) required the assumption that the function $f(y)$ satisfies $f^{\prime}(y) \geq k>0$ or $f(y) / y \geq$ $k>0$ for $y \neq 0$, (see 2, 5, 7-10,14,15,17, 18, 24), which is not applicable for $f(y)=|y|^{\nu-1} y$, the classical Emden-Fowler case. Very recently, the results of Atkinson [4] and Belohorec [6] for second order ordinary differential equation have been extended to (1.2) by Wong [21] under the assumption that the nonlinear function $f$ satisfies the sublinear condition

$$
0<\int_{0+}^{\varepsilon} \frac{d u}{f(u)}, \quad \int_{0-}^{-\varepsilon} \frac{d u}{f(u)}<\infty \quad \text { for all } \varepsilon>0,
$$

as well as the superlinear condition

$$
0<\int_{\varepsilon}^{\infty} \frac{d u}{f(u)}, \quad \int_{-\varepsilon}^{-\infty} \frac{d u}{f(u)}<\infty \quad \text { for all } \varepsilon>0 .
$$

Also it will be of great interest to find some oscillation criteria for the special case for (1.2), even for the Emden-Fowler equation

$$
(y(t)+p(t) y(t-\tau))^{\prime \prime}+q(t)|y(t-\sigma)|^{\nu-1} y(t-\sigma)=0, \quad \nu>0 .
$$


This problem was posed by Wong [21, Remark d]. As an affirmative answer to it, Saker [19], Saker and Manojlovic [20] have established some oscillation criteria for (1.2) and (1.3). However, these results cannot be applied to (1.1). Therefore, in the present paper, by using the averaging technique [13, 16, 22, 23], we shall establish several Kamenev-type oscillation criteria for (1.1). Our theorems essentially improve some known results in $[19,20]$. In particular, two interesting examples that point out the application of our results are also included.

\section{MAIN Results}

In this section, we shall establish Kamemev-type oscillation theorems for (1.1) under the cases when $0 \leq p(t) \leq 1$ and $-1<p_{0} \leq p(t) \leq 0$, which extend the results in $[13,16,22,23]$ to (1.1). It will be convenient to make the following notations in the remainder of this paper. Let $\phi \in C^{1}\left(\left[t_{0}, \infty\right), \mathbb{R}^{+}\right)$. Define

$$
\begin{aligned}
\mu & =\min \left\{\frac{\beta-\alpha}{\beta-\gamma}, \frac{\beta-\alpha}{\gamma-\alpha}\right\}, \quad g(s)=\phi(s) r\left(s-\sigma_{1}\right), \quad \theta=\frac{1}{(\gamma+1)^{\gamma+1}}, \\
Q_{1}(t) & =\mu \phi(t)\left[q_{1}^{\beta-\gamma}(t) q_{2}^{\gamma-\alpha}(t)\left(1-p\left(t-\sigma_{1}\right)\right)^{\alpha(\beta-\gamma)}\left(1-p\left(t-\sigma_{2}\right)\right)^{\beta(\gamma-\alpha)}\right]^{1 /(\beta-\alpha)}, \\
Q_{2}(t) & =\mu \phi(t)\left[q_{1}^{\beta-\gamma}(t) q_{2}^{\gamma-\alpha}(t)\right]^{1 /(\beta-\alpha)} .
\end{aligned}
$$

In order to present our theorems, we first introduce, following Philos [16], the function class $\Im$ which will be extensively used in the sequel. Namely, let $D_{0}=\left\{(t, s) \in \mathbb{R}^{2}: t>s \geq t_{0}\right\}$ and $D=\left\{(t, s) \in \mathbb{R}^{2}: t \geq s \geq t_{0}\right\}$. We will say that the function $H \in C(D, \mathbb{R})$ belongs to the class $\Im$, denoted by $H \in \Im$, if

( $H 1) H(t, t)=0$ for $t \geq t_{0}, H(t, s)>0$ on $(t, s) \in D_{0}$;

(H2) $H$ has a continuous and nonpositive partial derivative on $D_{0}$ with respect to the second variable such that

$$
\frac{\partial}{\partial s} H(r, s)=-h(t, s) H(t, s) \quad \text { for }(t, s) \in D_{0},
$$

where $h \in C(D, \mathbb{R})$.

For $\varphi \in C\left(\left[t_{0}, \infty\right), \mathbb{R}\right)$, we take an operator $A(\cdot ; T, t)$, which is defined in [22], in terms of $H$, as follows

$$
A(\varphi ; T, t)=\int_{T}^{t} H(r, s) \varphi(s) d s \text { for } t>T .
$$

It is easy to verify that $A(\cdot ; T, t)$ is an linear operator. 
For the sake of simplicity, throughout this paper, we always assume $H \in \Im, \phi \in$ $C^{1}\left(\left[t_{0}, \infty\right), \mathbb{R}^{+}\right)$, and the operator $A$ defined by (2.1) without further mentioning.

Theorem 2.1. (1.1) is oscillatory provided that one of the following conditions holds.

(1). $0 \leq p(t) \leq 1$, and

$$
\limsup _{t \rightarrow \infty} \frac{1}{H\left(t, t_{0}\right)} A\left(Q_{1}-\theta g\left|h-\phi^{\prime} \phi^{-1}\right|^{\gamma+1} ; t_{0}, t\right)=\infty .
$$

(2). $-1<p_{0} \leq p(t) \leq 0$, and

$$
\limsup _{t \rightarrow \infty} \frac{1}{H\left(t, t_{0}\right)} A\left(Q_{2}-\theta g\left|h-\phi^{\prime} \phi^{-1}\right|^{\gamma+1} ; t_{0}, t\right)=\infty .
$$

Proof. (1). Let $y(t)$ be a nonoscillatory solution of (1.1). Without loss of generality, we may assume that $y(t) \neq 0$ for $t \geq t_{0}$. Further, we suppose that there exists a $t_{1}>t_{0}$ such that

(2.4) $y(t)>0, \quad y(t-\tau)>0, \quad y\left(t-\sigma_{1}\right)>0$, and $y\left(t-\sigma_{2}\right)>0$ for $t \geq t_{1}$,

since the substitution $u=-y$ transforms (1.1) into an equation of the same form subject to the assumptions of Theorem. As an analogous proof of Lemma 1 (1) [24], see also [15], then, for some $T_{0} \geq t_{1}$, we have immediately that

$$
x(t)>0, \quad x^{\prime}(t)>0, \quad \text { and }\left(r(t)\left(x^{\prime}(t)\right)^{\gamma}\right)^{\prime} \geq 0 \text { for } t \geq T_{0} .
$$

Using (2.5), nothing that $x(t) \geq y(t)$, we have

$$
y(t)=x(t)-p(t) y(t-\tau) \geq x(t)-p(t) x(t-\sigma) \geq(1-p(t)) x(t) .
$$

Thus, for all $t>T_{0}$,

$y\left(t-\sigma_{1}\right) \geq\left(1-p\left(t-\sigma_{1}\right)\right) x\left(t-\sigma_{1}\right), \quad$ and $y\left(t-\sigma_{2}\right) \geq\left(1-p\left(t-\sigma_{2}\right)\right) x\left(t-\sigma_{2}\right)$.

Then (1.1) implies that

$$
\begin{aligned}
& \left(r(t)\left|x^{\prime}(t)\right|^{\gamma-1} x^{\prime}(t)\right)^{\prime}+q_{1}(t)\left(1-p\left(t-\sigma_{1}\right)\right)^{\alpha} x^{\alpha}\left(t-\sigma_{1}\right) \\
& \quad+q_{2}(t)\left(1-p\left(t-\sigma_{2}\right)\right)^{\beta} x^{\beta}\left(t-\sigma_{2}\right) \leq 0, \quad t \geq T_{0} .
\end{aligned}
$$

Define

$$
w(t)=\phi(t) \frac{r(t)\left|x^{\prime}(t)\right|^{\gamma-1} x^{\prime}(t)}{x^{\gamma}\left(t-\sigma_{1}\right)},
$$


clearly, $w(t)>0$. Differentiating (2.7) and using (2.6), we have

$$
\begin{aligned}
w^{\prime}(t) \leq & \frac{\phi^{\prime}(t)}{\phi(t)} w(t)-\phi(t)\left[q_{1}(t)\left(1-p\left(t-\sigma_{1}\right)\right)^{\alpha} x^{\alpha-\gamma}\left(t-\sigma_{1}\right)\right. \\
& \left.+q_{2}(t)\left(1-p\left(t-\sigma_{2}\right)\right)^{\beta} \frac{x^{\beta}\left(t-\sigma_{2}\right)}{x^{\gamma}\left(t-\sigma_{1}\right)}\right] \\
& -\frac{\gamma \phi(t) r(t)\left|x^{\prime}(t)\right|^{\gamma-1} x^{\prime}(t) x^{\prime}\left(t-\sigma_{1}\right)}{x^{\gamma+1}\left(t-\sigma_{1}\right)} .
\end{aligned}
$$

For simplicity, we consider the case $\sigma_{1} \geq \sigma_{2}$, (a similar argument holds for $\sigma_{1}<$ $\left.\sigma_{2}\right)$, then $x\left(t-\sigma_{1}\right) \leq x\left(t-\sigma_{2}\right)$. Hence, (2.8) yields that

$$
\begin{aligned}
w^{\prime}(t) \leq & \frac{\phi^{\prime}(t)}{\phi(t)} w(t)-\phi(t)\left[q_{1}(t)\left(1-p\left(t-\sigma_{1}\right)\right)^{\alpha} x^{\alpha-\gamma}\left(t-\sigma_{1}\right)\right. \\
& \left.+q_{2}(t)\left(1-p\left(t-\sigma_{2}\right)\right)^{\beta} x^{\beta-\gamma}\left(t-\sigma_{1}\right)\right]-\frac{\gamma w^{(\gamma+1) / \gamma}(t)}{g^{1 / \gamma}(t)},
\end{aligned}
$$

since $r(t)\left(x^{\prime}(t)\right)^{\gamma} \leq r\left(t-\sigma_{1}\right)\left(x^{\prime}\left(t-\sigma_{1}\right)\right)^{\gamma}$. The Young inequality [12, Theorem 61] implies that

$$
\begin{aligned}
& \frac{\beta-\gamma}{\beta-\alpha} q_{1}(t)\left(1-p\left(t-\sigma_{1}\right)\right)^{\alpha} x^{\alpha-\gamma}\left(t-\sigma_{1}\right)+\frac{\gamma-\alpha}{\beta-\alpha} q_{2}(t)\left(1-p\left(t-\sigma_{2}\right)\right)^{\beta} x^{\beta-\gamma}\left(t-\sigma_{1}\right) \\
& \geq\left[q_{1}^{\beta-\gamma}(t) q_{2}^{\gamma-\alpha}(t)\left(1-p\left(t-\sigma_{1}\right)\right)^{\alpha(\beta-\gamma)}\left(1-p\left(t-\sigma_{2}\right)\right)^{\beta(\gamma-\alpha)}\right]^{1 /(\beta-\alpha)} .
\end{aligned}
$$

Consequently,

$$
\begin{aligned}
& q_{1}(t)\left(1-p\left(t-\sigma_{1}\right)\right)^{\alpha} x^{\alpha-\gamma}\left(t-\sigma_{1}\right)+q_{2}(t)\left(1-p\left(t-\sigma_{2}\right)\right)^{\beta} x^{\beta-\gamma}\left(t-\sigma_{2}\right) \\
& \quad \geq \mu\left[q_{1}^{\beta-\gamma}(t) q_{2}^{\gamma-\alpha}(t)\left(1-p\left(t-\sigma_{1}\right)\right)^{\alpha(\beta-\gamma)}\left(1-p\left(t-\sigma_{2}\right)\right)^{\beta(\gamma-\alpha)}\right]^{1 /(\beta-\alpha)} .
\end{aligned}
$$

Combining (2.9) and (2.10), we have

$$
w^{\prime}(t) \leq-Q_{1}(t)+\frac{\phi^{\prime}(t)}{\phi(t)} w(t)-\frac{\gamma}{g^{1 / \gamma}(t)} w^{(\gamma+1) / \gamma}(t), \quad t \geq T_{0} .
$$

Apply the operator $A(\cdot ; T, t), t>T \geq T_{0}$, to (2.11), and use $(H 2)$ to find

$$
\begin{aligned}
A\left(Q_{1} ; T, t\right) \leq & H(t, T) w(T)+A\left(\left|h-\phi^{\prime} \phi^{-1}\right| w ; T, t\right) \\
& -\gamma A\left(g^{-1 / \gamma} w^{(\gamma+1) / \gamma} ; T, t\right) .
\end{aligned}
$$

For given $t$ and $s,(t \neq s)$, set

$$
F(u):=\left|h-\phi^{\prime} \phi^{-1}\right| u-\frac{\gamma}{g^{1 / \gamma}} u^{(\gamma+1) / \gamma}, \quad u>0 .
$$


$F(u)$ attains its maximum at

$$
u=\frac{g}{(\gamma+1)^{\gamma}}\left|h-\phi^{\prime} \phi^{-1}\right|^{\gamma}
$$

and

$$
F(u) \leq F_{\max }=\theta g\left|h-\phi^{\prime} \phi^{-1}\right|^{\gamma+1} .
$$

Then by using (2.13) in (2.12), we get

$$
A\left(Q_{1} ; T, t\right) \leq H(t, T) w(T)+\theta A\left(g\left|h-\phi^{\prime} \phi^{-1}\right|^{\gamma+1} ; T, t\right) .
$$

Set $T=T_{0}$ and in view of $(H 2)$, so

$$
A\left(Q_{1}-\theta g\left|h-\phi^{\prime} \phi^{-1}\right|^{\gamma+1} ; T_{0}, t\right) \leq H\left(t, T_{0}\right) w\left(T_{0}\right) \leq H\left(t, t_{0}\right) w\left(T_{0}\right) .
$$

Thus, by $(H 2)$, we obtain

$$
\begin{aligned}
A\left(Q_{1}-\theta g\left|h-\phi^{\prime} \phi^{-1}\right|^{\gamma+1} ; t_{0}, t\right) & \\
= & A\left(Q_{1}-\theta g\left|h-\phi^{\prime} \phi^{-1}\right|^{\gamma+1} ; t_{0}, T_{0}\right) \\
& +A\left(Q_{1}-\theta g\left|h-\phi^{\prime} \phi^{-1}\right|^{\gamma+1} ; T_{0}, t\right) \\
\leq & H\left(t, t_{0}\right)\left(\int_{t_{0}}^{T_{0}} Q_{1}(s) d s+w\left(T_{0}\right)\right) .
\end{aligned}
$$

Divide (2.15) through by $H\left(t, t_{0}\right)$ and take limsup in it as $t \rightarrow \infty$. Condition (2.2) gives a desired contradiction in (2.15). This proves Case (1).

(2). Let $y(t)$ be a nonoscillatory solution of (1.1). Without loss of generality, we may assume that $y(t) \neq 0$ for $t \geq t_{0}$. Further, we suppose that there exists a $t_{1}>t_{0}$ such that (2.4) holds. As an analogous proof of Lemma 1 (2) [24]. Then, for some $T_{0} \geq t_{1}$, we still have that (2.5) holds for $t \geq T_{0}$. Noting that $y(t) \geq x(t)$, we get

$$
y\left(t-\sigma_{1}\right) \geq x\left(t-\sigma_{1}\right), y\left(t-\sigma_{2}\right) \geq x\left(t-\sigma_{2}\right) \text { for } t \geq T_{0} .
$$

Then, (1.1) changes into

$$
\left(r(t)\left|x^{\prime}(t)\right|^{\gamma-1} x^{\prime}(t)\right)^{\prime}+q_{1}(t) x^{\alpha}\left(t-\sigma_{1}\right)+q_{2}(t) x^{\beta}\left(t-\sigma_{2}\right) \leq 0, \quad t \geq T_{0} .
$$

Consider the function $w(t)$ defined by (2.7), similar to the proof of (2.9), we can obtain

$$
\begin{aligned}
w^{\prime}(t) \leq & \frac{\phi^{\prime}(t)}{\phi(t)} w(t)-\phi(t)\left[q_{1}(t) x^{\alpha-\gamma}\left(t-\sigma_{1}\right)+q_{2}(t) x^{\beta-\gamma}\left(t-\sigma_{1}\right)\right] \\
& -\frac{\gamma}{g^{1 / \gamma}(t)} w^{(\gamma+1) / \gamma}(t) .
\end{aligned}
$$


The rest of the proof is similar to that of Case (1), hence, is omitted.

Theorem 2.2. (1.1) is oscillatory provided that one of the following conditions holds.

(3). $0 \leq p(t) \leq 1$, and there exist $\varphi_{1}, \varphi_{2} \in C\left(\left[t_{0}, \infty\right), \mathbb{R}\right)$ such that for all $T \geq t_{0}$,

$$
\begin{gathered}
\limsup _{t \rightarrow \infty} \frac{1}{H(t, T)} A\left(Q_{1} ; T, t\right) \geq \varphi_{1}(T), \\
\limsup _{t \rightarrow \infty} \frac{1}{H(t, T)} A\left(g\left|h-\phi^{\prime} \phi^{-1}\right|^{\gamma+1} ; T, t\right) \leq \varphi_{2}(T),
\end{gathered}
$$

where $\varphi_{1}$ and $\varphi_{2}$ satisfy

$$
\liminf _{t \rightarrow \infty} \frac{1}{H(t, T)} A\left(g^{-1 / \gamma}\left(\varphi_{1}-\theta \varphi_{2}\right)_{+}^{(\gamma+1) / \gamma} ; T, t\right)=\infty .
$$

(4). $-1<p_{0} \leq p(t) \leq 0$, and there exist $\varphi_{1}, \varphi_{2} \in C\left(\left[t_{0}, \infty\right), \mathbb{R}\right)$ such that for all $T \geq t_{0}$,

$$
\limsup _{t \rightarrow \infty} \frac{1}{H(t, T)} A\left(Q_{2} ; T, t\right) \geq \varphi_{1}(T),
$$

and (2.18) hold, where $\varphi_{1}$ and $\varphi_{2}$ satisfy (2.19), and $\varphi_{+}=\max \{0, \varphi\}$.

Proof. We only show Case (3). The proof of Case (4) is similar to that of Case (3). Proceeding as the proof of Case (1) of Theorem 2.1, we have (2.12) and (2.14) hold, again divide (2.14) by $H(t, T)$, and obtain

(2.21) $\frac{1}{H(t, T)} A\left(Q_{1} ; T, t\right)-\frac{\theta}{H(t, T)} A\left(g\left|h-\phi^{\prime} \phi^{-1}\right|^{\gamma+1} ; T, t\right) \leq w(T), \quad t>T$.

Take limsup in (2.21) as $t \rightarrow \infty$, and note from (2.17) and (2.18) that

$$
\varphi_{1}(T)-\theta \varphi_{2}(T) \leq w(T)
$$

from which it follows that

$$
\begin{aligned}
& \frac{1}{H(t, T)} A\left(g^{-1 / \gamma}\left(\varphi_{1}-\theta \varphi_{2}\right)_{+}^{(\gamma+1) / \gamma} ; T, t\right) \\
& \quad \leq \frac{1}{H(t, T)} A\left(g^{-1 / \gamma} w^{(\gamma+1) / \gamma} ; T, t\right) .
\end{aligned}
$$


On the other hand, by (2.12), we have

$$
\begin{aligned}
\frac{\gamma}{H(t, T)} A\left(g^{-1 / \gamma} w^{(\gamma+1) / \gamma} ; T, t\right)- & \frac{1}{H(t, T)} A\left(\left|h-\phi^{\prime} \phi^{-1}\right| w ; T, t\right) \\
& \leq w(T)-\frac{1}{H(t, T)} A\left(Q_{1} ; T, t\right) .
\end{aligned}
$$

Thus, by (2.17),

$$
\begin{aligned}
\liminf _{r \rightarrow \infty} & \left\{\frac{\gamma}{H\left(t, T_{0}\right)} A\left(g^{-1 / \gamma} w^{(\gamma+1) / \gamma} ; T_{0}, t\right)\right. \\
& \left.-\frac{1}{H\left(t, T_{0}\right)} A\left(\left|h-\phi^{\prime} \phi^{-1}\right| w ; T_{0}, t\right)\right\} \leq w\left(T_{0}\right)-\varphi_{1}\left(T_{0}\right) \leq C_{0},
\end{aligned}
$$

where $C_{0}$ is a constant. According to (2.23), there exists a sequence $\left\{t_{j}\right\}_{j=1}^{\infty} \in$ $\left[t_{0}, \infty\right)$ with $\lim _{j \rightarrow \infty} t_{j}=\infty$ such that

$$
\begin{aligned}
& \frac{\gamma}{H\left(t_{j}, T_{0}\right)} A\left(g^{-1 / \gamma} w^{(\gamma+1) / \gamma} ; T_{0}, t_{j}\right) \\
& \quad-\frac{1}{H\left(t_{j}, T_{0}\right)} A\left(\left|h-\phi^{\prime} \phi^{-1}\right| w ; T_{0}, t_{j}\right) \leq C_{0}+1 .
\end{aligned}
$$

Now, we claim that

$$
\liminf _{t \rightarrow \infty} \frac{1}{H\left(t, T_{0}\right)} A\left(g^{-1 / \gamma} w^{(\gamma+1) / \gamma} ; T_{0}, t\right)<\infty .
$$

If (2.25) does not hold, and noting that (2.24), we get

$$
\lim _{j \rightarrow \infty} \frac{1}{H\left(r_{j}, T_{0}\right)} A\left(g^{-1 / \gamma} w^{(\gamma+1) / \gamma} ; T_{0}, t_{j}\right)=\infty .
$$

So, (2.24) and (2.26) gives, for $j$ large enough,

$$
\frac{A\left(\left|h-\phi^{\prime} \phi^{-1}\right| w ; T_{0}, t_{j}\right)}{A\left(g^{-1 / \gamma} w^{(\gamma+1) / \gamma} ; T_{0}, t_{j}\right)}-\gamma \geq-\frac{\gamma}{2},
$$

that is,

$$
A\left(\left|h-\phi^{\prime} \phi^{-1}\right| w ; T_{0}, t_{j}\right) \geq \frac{\gamma}{2} A\left(g^{-1 / \gamma} w^{(\gamma+1) / \gamma} ; T_{0}, t_{j}\right) .
$$

The Hölder inequality follows

$$
\begin{aligned}
A\left(\left|h-\phi^{\prime} \phi^{-1}\right| w ; T_{0}, t_{j}\right) \leq & \left(A\left(g^{-1 / \gamma} w^{(\gamma+1) / \gamma} ; T_{0}, t_{j}\right)\right)^{\gamma /(\gamma+1)} \\
& \left(A\left(g\left|h-\phi^{\prime} \phi^{-1}\right|^{\gamma+1} ; T_{0}, t_{j}\right)\right)^{1 /(\gamma+1)} .
\end{aligned}
$$


From (2.27) and (2.28), we obtain

$$
\begin{aligned}
\left(\frac{\gamma}{2}\right)^{\gamma+1} & \frac{1}{H\left(t_{j}, T_{0}\right)} A\left(g^{-1 / \gamma} w^{(\gamma+1) / \gamma} ; T_{0}, t_{j}\right) \\
& \leq \frac{1}{H\left(t_{j}, T_{0}\right)} A\left(g\left|h-\phi^{\prime} \phi^{-1}\right|^{(\gamma+1) / \gamma} ; T_{0}, t_{j}\right) .
\end{aligned}
$$

By (2.18), the right-hand side of (2.29) is bounded, which contradicts (2.26). Thus (2.25) holds. Hence, by (2.22),

$$
\begin{aligned}
\liminf _{r \rightarrow \infty} & \frac{1}{H(t, T)} A\left(g^{-1 / \gamma}\left(\varphi_{1}-\theta \varphi_{2}\right)_{+}^{(\gamma+1) / \gamma} ; T, t\right) \\
& \leq \liminf _{r \rightarrow \infty} \frac{1}{H(t, T)} A\left(g^{-1 / \gamma} w^{(\gamma+1) / \gamma} ; T, t\right)<\infty .
\end{aligned}
$$

which contradicts (2.19).

Remark 2.1. Let $\gamma=1$. For the superlinear equation (1.2), Theorems 2.1-2.2 improve the main results in [20]. For the Emden-Fowler type equation (1.3), our results extend and improve the main results in [19].

\section{Corollaries AND EXAMPLES}

As Theorems 2.1 and 2.2 are rather general, it is convenient for applications to derive a number of oscillation criteria with the appropriate choice of the functions $H$ and $\phi$. In this section, we will give some corollaries of Theorems 2.1 and 2.2, and show the applications of our main results in two interesting examples. Further, we will see that equations (3.10) and (3.11) are oscillatory based on Corollary 3.2 and Theorem 2.2, though the known results such as in [1-3,7-10,14,15,17-21,24] fail to apply to these equations.

As an immediate consequence of Theorem 2.1, we have the following corollary.

Corollary 3.1. (1.1) is oscillatory provided that one of the following conditions holds.

(5). $0 \leq p(t) \leq 1$, and

$$
\limsup _{t \rightarrow \infty} \frac{1}{H\left(t, t_{0}\right)} A\left(Q_{1} ; t_{0}, t\right)=\infty,
$$

and

$$
\limsup _{t \rightarrow \infty} \frac{1}{H\left(t, t_{0}\right)} A\left(g\left|h-\phi^{\prime} \phi^{-1}\right|^{\gamma+1} ; t_{0}, t\right)<\infty .
$$


(6). $-1<p_{0} \leq p(t) \leq 0$, and

$$
\limsup _{t \rightarrow \infty} \frac{1}{H\left(t, t_{0}\right)} A\left(Q_{2} ; t_{0}, t\right)=\infty
$$

and

$$
\limsup _{t \rightarrow \infty} \frac{1}{H\left(t, t_{0}\right)} A\left(g\left|h-\phi^{\prime} \phi^{-1}\right|^{\gamma+1} ; t_{0}, t\right)<\infty
$$

Corollary 3.1. (1.1) is oscillatory provided that one of the following conditions holds.

(7). $0 \leq p(t) \leq 1$, and for some $\lambda>\gamma$,

$$
\limsup _{t \rightarrow \infty} \frac{1}{G^{\lambda}(t)} \int_{t_{0}}^{t}[G(t)-G(s)]^{\lambda} Q_{3}(s) d s=\infty .
$$

(8). $-1<p_{0} \leq p(t) \leq 0$, and for some $\lambda>\gamma$,

$$
\limsup _{t \rightarrow \infty} \frac{1}{G^{\lambda}(t)} \int_{t_{0}}^{t}[G(t)-G(s)]^{\lambda} Q_{4}(s) d s=\infty .
$$

Where

$$
\begin{aligned}
G(t) & =\int_{t_{0}}^{t} \frac{d s}{r^{1 / \gamma}\left(s-\sigma_{1}\right)}, \\
Q_{3}(t) & =\mu\left[q_{1}^{\beta-\gamma}(t) q_{2}^{\gamma-\alpha}(t)\left(1-p\left(t-\sigma_{1}\right)\right)^{\alpha(\beta-\gamma)}\left(1-p\left(t-\sigma_{2}\right)\right)^{\beta(\gamma-\alpha)}\right]^{1 /(\beta-\alpha)}, \\
Q_{4}(t) & =\mu\left[q_{1}^{\beta-\gamma}(t) q_{2}^{\gamma-\alpha}(t)\right]^{1 /(\beta-\alpha)} .
\end{aligned}
$$

Proof. Let

$$
H(t, s)=[G(t)-G(s)]^{\lambda} \quad \text { and } \quad \phi(t)=1,
$$

then

$$
h(t, s)=\frac{\lambda}{r^{1 / \gamma}\left(s-\sigma_{1}\right)[G(t)-G(s)]} .
$$

Note that 


$$
A\left(g\left|h-\phi^{\prime} \phi^{-1}\right|^{\gamma+1} ; T, t\right)=\frac{\lambda^{\gamma+1}}{\lambda-\gamma}\left[G^{\lambda-\gamma}(t)-G^{\lambda-\gamma}(T)\right],
$$

it is easy to show that Corollary 3.2 holds from Corollary 3.1 .

By Theorem 2.2, we have

Corollary 3.3. Let $\lim _{t \rightarrow \infty} G(t)=\infty$. Then (1.1) is oscillatory provided that one of the following conditions holds.

(9). $0 \leq p(t) \leq 1$, and there exists $\varphi \in C\left(\left[t_{0}, \infty\right), \mathbb{R}\right)$ such that for some $\lambda>\gamma$ and all $T \geq t_{0}$,

$$
\limsup _{t \rightarrow \infty} \frac{1}{G^{\lambda}(t)} \int_{T}^{t}[G(t)-G(s)]^{\lambda} Q_{3}(s) d s \geq \varphi(T),
$$

where $\varphi$ satisfies

$$
\liminf _{t \rightarrow \infty} \frac{1}{G^{\lambda}(t)} \int_{T}^{t}[G(t)-G(s)]^{\lambda} g^{-1 / \gamma}(s) \varphi^{(\gamma+1) / \gamma}(s) d s=\infty .
$$

(10). $-1<p_{0} \leq p(t) \leq 0$, and there exists $\varphi \in C\left(\left[t_{0}, \infty\right), \mathbb{R}\right)$ such that (3.8) holds and for some $\lambda>\gamma$ and all $T \geq t_{0}$,

$$
\limsup _{t \rightarrow \infty} \frac{1}{G^{\lambda}(t)} \int_{T}^{t}[G(t)-G(s)]^{\lambda} Q_{4}(s) d s \geq \varphi(T),
$$

where $Q_{3}(t), Q_{4}(t)$ and $G(t)$ are defined in Corollary 3.2.

Remark 3.1. Following the classical ideas of Kamenev [13], we define $H(t, s)$ as

$$
H(t, s)=(t-s)^{\lambda}, \quad(t, s) \in D,
$$

where $\lambda>\gamma$. Therefore, as a consequence of Theorems 2.1 and 2.2, we can obtain two oscillation criteria. Here, we omit the details.

The following two examples illustrate our main results

Example 3.1. Consider the following neutral differential equation

$$
\begin{aligned}
& \left(\left|x^{\prime}(t)\right| x^{\prime}(t)\right)^{\prime}+q_{1}(t)|y(t-3)|^{1 /(2 n+1)} y(t-3) \\
& +q_{2}(t)|y(t-2)|^{(4 n+1) /(2 n+1)} y(t-2)=0, \quad t \geq 3,
\end{aligned}
$$


where $r(t)=1, x(t)=y(t)+p(t) x(t-1), p(t)=p / \sqrt{t+3}, p= \pm 1, \gamma=2$, $\alpha=(2 n+2) /(2 n+1), \beta=(6 n+2) /(2 n+1), n$ is positive integer, and $q_{1}, q_{2} \in$ $C\left([3, \infty), \mathbb{R}^{+}\right)$with $q_{1}(t) q_{2}(t) \geq \lambda_{1}^{2} / t^{2},\left(\lambda_{1}>0\right)$.

Now, we consider the following two cases.

Case 1. $p=1$. Noting that $p(t-3)>p(t-2)$, we have

$$
\mu=2, \quad G(t)=t-3, \quad \text { and } \quad Q_{3}(t) \geq \frac{2 \lambda_{1}}{t}\left(1-\frac{1}{\sqrt{t}}\right)^{2} .
$$

Then, by Theorem 41 in [12],

$$
(t-s)^{\lambda} \geq t^{\lambda}-\lambda s t^{\lambda-1} \quad \text { for } \lambda>1, t \geq s \geq 1,
$$

we have, for $\lambda>\gamma$,

$$
\begin{aligned}
& \limsup _{t \rightarrow \infty} \frac{1}{G^{\lambda}(t)} \int_{t_{0}}^{t}[G(t)-G(s)]^{\lambda} Q_{3}(s) d s \\
& \geq \limsup _{t \rightarrow \infty} \frac{2 \lambda_{1}}{t^{\lambda}} \int_{3}^{t} \frac{(t-s)^{\lambda}}{s}\left(1-\frac{1}{\sqrt{s}}\right)^{2} d s \\
& \quad \geq 2 \lambda_{1}\left(1-\frac{1}{\sqrt{3}}\right)^{2} \limsup _{t \rightarrow \infty} \frac{1}{t^{\lambda}} \int_{3}^{t} \frac{t^{\lambda}-\lambda t^{\lambda-1} s}{s} d s=\infty .
\end{aligned}
$$

Hence, by Corollary 3.2 (7), (3.10) is oscillatory.

Case 2. $p=-1$. We have

$$
\mu=2, \quad G(t)=t-3, \quad \text { and } \quad Q_{4}(t) \geq \frac{2 \lambda_{1}}{t} .
$$

The rest of proof is similar to that of Case 1. Hence (3.10) is oscillatory by Corollary $3.2(8)$.

Example 3.2. Consider the following neutral differential equation

$$
\begin{gathered}
\left(\left|x^{\prime}(t)\right|^{\gamma-1} x^{\prime}(t)\right)^{\prime}+q_{1}(t)|y(t-3)|^{\alpha-1} y(t-3) \\
+q_{2}(t)|y(t-2)|^{\beta-1} y(t-2)=0, \quad t \geq 3,
\end{gathered}
$$

where $x(t)=y(t)+p y(t-1),-1<p<1, r(t)=1,0<\alpha<\gamma<\beta$ with $\gamma=(\alpha+\beta) / 2$, and $q_{1}, q_{2} \in C\left([3, \infty), \mathbb{R}^{+}\right)$with $q_{1}(t) q_{2}(t) \geq\left(\lambda_{2} t^{\gamma-1}\right)^{2},\left(\lambda_{2}>0\right)$. 
For Theorem 2.2, let $\phi(t)=t^{-(\gamma+1)}$. Now, we consider the following two cases.

Case 1. $0 \leq p<1$. A direct computation yields

$$
\mu=2, \quad g(t)=t^{-(\gamma+1)}, \quad \text { and } \quad Q_{1}(t) \geq \frac{2 \lambda_{2}(1-p)^{\gamma}}{t^{2}} .
$$

Let $H(t, s)=(t-s)^{\gamma+1}$, then $h(t, s)=(\gamma+1) /(t-s)$. Thus, for all $t>T \geq 3$,

$$
\begin{aligned}
& \limsup _{t \rightarrow \infty} \frac{1}{H(t, T)} A\left(Q_{1} ; T, t\right)=\limsup _{t \rightarrow \infty} \frac{1}{(t-T)^{\gamma+1}} \int_{T}^{t}(t-s)^{\gamma+1} Q_{1}(s) d s \\
& \geq 2 \lambda_{2}(1-p)^{\gamma} \limsup _{t \rightarrow \infty} \frac{1}{(t-T)^{\gamma+1}} \int_{T}^{t} \frac{(t-s)^{\gamma+1}}{s^{2}} d s \\
& \quad=2 \lambda_{2}(1-p)^{\gamma} \limsup _{t \rightarrow \infty} \frac{1}{(t-T)^{\gamma+1}} \int_{T}^{t} \frac{t^{\gamma+1}-(\lambda+1) t^{\gamma} s}{s^{2}} d s \\
& =\frac{2 \lambda_{2}(1-p)^{\gamma}}{T}
\end{aligned}
$$

and

$$
\begin{aligned}
& \limsup _{t \rightarrow \infty} \frac{1}{H(t, T)} A\left(g\left|h-\phi^{\prime} \phi^{-1}\right|^{\gamma+1} ; T, t\right) \\
& \quad=\limsup _{t \rightarrow \infty} \frac{(\gamma+1)^{\gamma+1}}{(t-T)^{\gamma+1}} \int_{T}^{t} \frac{t^{\gamma+1}}{s^{2(\gamma+1)}} d s=\frac{(\gamma+1)^{\gamma+1}}{(2 \gamma+1) T^{2 \gamma+1}} .
\end{aligned}
$$

Set,

$$
\varphi_{1}(T)=\frac{2 \lambda_{2}(1-p)^{\gamma}}{T} \text { and } \varphi_{2}(T)=\frac{(\gamma+1)^{\gamma+1}}{2 \gamma+1} \frac{1}{T^{2 \gamma+1}} .
$$

Thus, for $\lambda_{2}>(9(1-p))^{-\gamma} /(4 \gamma+2)$,

$$
\begin{aligned}
& \limsup _{t \rightarrow \infty} \frac{1}{H(t, T)} A\left(g^{-1 / \gamma}\left(\varphi_{1}-\theta \varphi_{2}\right)_{+}^{\gamma+1} ; T, t\right) \\
& \quad=\liminf _{t \rightarrow \infty} \frac{1}{(t-T)^{\gamma+1}} \int_{T}^{t}(t-s)^{\gamma+1}\left(2 \lambda_{2}(1-p)^{\gamma}-\frac{1}{(2 \gamma+1) s^{2 \gamma}}\right)^{(\gamma+1) / \gamma} d s \\
& \quad \geq\left(2 \lambda_{2}(1-p)^{\gamma}-\frac{1}{9^{\gamma}(2 \gamma+1)}\right)^{(\gamma+1) / \gamma} \liminf _{t \rightarrow \infty} \frac{1}{(t-T)^{\gamma+1}} \int_{T}^{t}(t-s)^{\gamma+1} d s=\infty
\end{aligned}
$$

It follows from Theorem 2.2 (3) that (2.11) is oscillatory for $\lambda_{2}>(9(1-p))^{-\gamma} /(4 \gamma+$ $2)$. 
Case 2. $-1 \leq p \leq 0$. Then

$$
\mu=2, \quad g(t)=t^{-(\gamma+1)}, \quad Q_{2}(t) \geq \frac{2 \lambda_{2}}{t^{2}} .
$$

The rest of proof is similar to that of Case 1. Hence, by Theorem 2.2 (4), (3.11) is oscillatory for $\lambda_{2}>9^{-\gamma} /(4 \gamma+2)$.

\section{ACKNOWLEDGMENT}

The author would like to express his great appreciation to the referees for their careful reading of the manuscript and correcting many grammatical mistakes and for their helpful suggestions.

\section{REFERENCES}

1. S. H. Abdallah, Oscillatory and non-oscillatory behavior of second order neutral delay differential equations, Appl. Math. Comput., 135 (2003), 333-344.

2. R. P. Agarwal, S. R. Grace and D. O'Regan, Oscillation Theory for Second Order Dynamic Equations, Taylor \& Francis, Laondon, 2003.

3. R. P. Agarwal, S. R. Grace and D. O'Regan, Nonoscillatory solutions of delay and neutral singular differential equations, Appl. Anal., 81 (2002), 1221-1244.

4. F. V. Atkinson, On second order nonlinear oscillation, Pacific J. Math., 5 (1955), 643-647.

5. D. D. Bainov and D. P. Mishev, Oscillation Theory for Neutral Equations with delay, Adam Hilger IOP Publishing Ltd., New York, 1991.

6. S. Belohorec, Oscillatory solutions of certain nonlinear differential equations of the second order, Math. Fyz. Casopis Sloven Akad. Vied., 11 (1961), 250-255.

7. J. Dzurina and B. Mihalikova, Oscillation criteria for second order neutral differential equations, Math. Boh., 125 (2000), 145-153.

8. L. H. Erbe, Q. Kong and B. G. Zhang, Oscillation Theory for Functional Differential Equations, Marcel Dekker, New York, 1995.

9. M. K. Grammatikopoulos, G. Ladas and A. Meimaidou, Oscillation of second order neutral delay differential equations, Radovi Mat., 1 (1985), 267-274.

10. I. Györi and G. Ladas, Oscillation Theory of Delay Differential Equations With Applications, Clarendou Press, Oxford, 1991.

11. J. K. Hale, Theory of Functional Differential Equations, Springer-Verlag, New York, 1977.

12. G. H. Hardy, J. E. Littlewood and G. Polya, Inequalities, 2nd ed, Cambridge University Press, 1988. 
13. I. V. Kamenev, An integral criterion for oscillation of linear differential equations, Mat. Zamtki. (in Russian), 23 (1978), 249-251.

14. H. J. Li, Oscillation theorems for second order neutral delay differential equations, Nonlinear Anal. TMA., 26 (1996), 1397-1409.

15. H. J. Li and W. L. Liu, Oscillation criteria for second order neutral differential equations, Canada J. Math., 48 (1996), 871-886.

16. Ch. G. Philos, Oscillation theorems for linear differential equation of second order, Arch. Math (Basel)., 53 (1989), 482-492.

17. S. G. Ruan, Oscillations of second order neutral differential equations, Canad. Math. Bull., 36 (1993), 485-496.

18. Y. Sahiner, On oscillation of second order neutral type delay differential equations, Appl. Math. Comput., 150 (2004), 697-706.

19. S. H. Saker, Oscillation for second order neutral delay differential equations of Emden-Fowler type, Acta Math. Hungar., 100(1-2) (2003), 37-62.

20. S. H. Saker and J. V. Manojlović, Oscillation criteria for second order superlinear neutral delay differential equations, Elect. J. Qualit. Theory of Diff. Eqs., 10 (2004), $1-22$.

21. J. S. W. Wong, Necessary and sufficient conditions for oscillation for second order neutral differential equations, J. Math. Anal. Appl., 252 (2000), 342-352.

22. J. S. W. Wong, On Kamenev-tye oscillation theorems for second order differential equations, J. Math. Anal. Appl., 258 (2001), 244-257.

23. J. Yan, Oscillation theorems for second order linear differential equations with damping, Proc. Amer. Math. Soc., 98 (1980), 276-282.

24. Q. Yang, L. Yang and S. Zhu, Interval criteria for oscillation of second order nonlinear neutral differential equations, Comput. Math. Appl., 46 (2003), 903-918.

Zhiting $\mathrm{Xu}$

School of Mathematical Sciences,

South China Normal University,

Guangzhou, 510631,

P. R. China

E-mail: xztxhyyj@pub.guangzhou.gd.cn 